\title{
Identifying key factors of the transmission dynamics of drug-resistant malaria
}

\author{
Tamsin E. Lee ${ }^{\mathrm{a}, \mathrm{b}}$, Melissa A. Penny ${ }^{\mathrm{a}, \mathrm{b}}$ \\ ${ }^{a}$ Department of Epidemiology and Public Health, Swiss Tropical and Public Health \\ Institute, Basel, Switzerland \\ ${ }^{b}$ University of Basel, Basel, Switzerland
}

\begin{abstract}
Development of resistance to malaria treatments remains a great threat to continued malaria burden reduction and elimination. Quantifying the impact of key factors which increase the emergence and spread of drug resistance can guide intervention strategies. Whilst modelling provides a framework to understand these factors, we show that a simple of model with a sensitiveresistant dichotomy leads to incorrectly focusing on reducing the treatment rate as a means to prevent resistance. Instead we present a model that considers the development of resistance within hosts as a scale, and we then quantify the number of resistant infections that would arise from a single sensitive infection. By including just one step before full resistance, the model highlights that disrupting this development is more effective than reducing treatment rate. This result is compounded when the model includes the more realistic scenario of several intermediary steps. An additional comparison to transmission probabilities, where resistant infections are less likely to be transmitted (cost of resistance), confirms that preventing the establishment of resistance is more effective than controlling the spread. Our work strongly advocates for further studies into within-host models of resistance, including the potential of combination therapies to disrupt emergence.
\end{abstract}

Keywords: Ross-MacDonald model, malaria treatment, resistance emergence, resistance spread, resistance establishment, mutation

\section{Introduction}

Resistance threatens not just control of malaria but also our potential to 3 eliminate malaria in low prevalence settings. Several historical examples of 
development of the spread of resistance to malaria treatment exist, such as widespread chloroquine resistance and less geographically spread sulfadoxine/pyrimethamine resistance, and more recently, resistance to artemisinin (Yeung, 2004, WHO, 2018). Even once a drug is no longer in use, the resistant genotypes may decline slowly, or even persist indefinitely (Liechti et al. 2017).

In the early 2000s combination therapy, where an infected individual is treated with two or more drugs, became accepted as an approach to prevent resistance to a given particular drug given as monotherapy (World Health Organization 2001). Artemisinin combination therapies were introduced with short-acting artemisinin derivatives formulated with different longer-acting partner drugs, such lumefantrine or Mefloquine (Nosten and White 2007). Nonetheless, resistance continues to occur, with artemisinin resistance developing in South East Asia (Ménard et al. 2016) with fear of further spreading and thus threatening both morbidity control and elimination of malaria. More recently triple combination therapies with the view to delay emergence and spread are being tested (Shanks et al. 2014). The community state that to eliminate malaria policy decisions need to be preemptive, not reactive (Boni et al. 2016). This requires a deeper understanding of resistance which cannot be gained from generalisations of specific case studies. To test and understand key drivers of resistance, mathematical models provide an invaluable framework.

zur Wiesch et al. (2011) consider the overall dynamics, and discuss which factors could influence the growth of resistance, including mutation, recombination and de novo versus transmitted resistance. Typically reducing the probability that de novo resistance mutations occur is often the focus, meaning that pathogens are rapidly eliminated and patients continue treatment after they feel better. However, Read et al. (2011) argue that the more aggressive the regime, the greater the selection pressure in favour of resistance. Essentially, reducing resistance is a balance between reducing the probability of de novo resistance whilst not creating opportunity for mutated genotypes to grow rapidly (Day and Read 2016). Kouyos et al. (2014) relate this balance to high and low transmission areas, advising moderate treatment where malaria has high-transmission since co-infection is more frequent, and thus resistant and sensitive strains compete more often within an individual host. Aggressive treatment would be more likely to cause the removal of a sensitive competitor. In a summary of population genetics and epidemiological models for drug resistance, Mackinnon (2005) states that the two overriding 
factors are the proportion of humans treated with drugs, and the efficacy of the drug in clearing parasites.

When drug concentration is low enough to kill the sensitive genotype, it may not necessarily be high enough to kill partially-resistant mutations. With more uninfected blood cells, partially-resistant and resistant genotypes can multiply rapidly. This selection process is often summarised by the selection coefficient, which is simply the difference between the growth rate of the mutant type and the sensitive type for a given drug concentration - the relative fitness (Huijben et al. 2011). So a large selection coefficient implies that the mutant type is growing rapidly. Day et al. (2015) contend that instead of the relative fitness, the absolute fitness is a better measure. That is, the growth rate of the mutant type is compared to itself at a baseline rate defined by both the drug concentration and a within patient state variable, such as the density of resources, or immune cells.

Having established that a resistant infection develops within a host, the transmission of this infection throughout the homogeneous population can be modelled via a compartment model. The most well-known example of a compartmental model for malaria transmission is the Ross-MacDonald model (Ross 1911, Macdonald 1957, Dietz 1974). This model puts the main burden of transmission on mosquito-specific features, and thus motivated mosquitobased malaria control programmes (Mandal et al. 2011). The simplicity and relevance of the Ross-MacDonald has ensured that it continues to be a strong basis for a broader theory of mosquito-bourne disease transmission and control (Smith et al. 2012).

There are several compartmental models that include a treated population, and a population resistant to treatment. We compare our model to six models which we are aware of, see Table 1. Two of the models, Koella and Antia (2003) and Chiyaka et al. (2009), include an immune population. As expected, the three models which explicitly include a treated population (Koella and Antia (2003), Esteva et al. (2009) and Chiyaka et al. (2009)) find that the proportion treated has an effect on the spread of drug resistance. The models of Koella and Antia (2003) and Esteva et al. (2009) also find that the spread of resistant infections depends on the effectiveness of the treatment (defined in terms of the period of infection), and the cost of resistance (defined in terms of the reduction of intensity of transmission due to mutation). Their models do not indicate transmission as a significant factor. Chiyaka et al. (2009) also show that the spread of drug resistance depends on the infectious periods, defined here as the ratio of the infectious 
periods of treated and untreated humans. Unlike Koella and Antia (2003) and Esteva et al. (2009), Chiyaka et al. (2009) find the transmission rates from infectious humans with resistant and sensitive infections to influence the spread of resistant infections. Tumwiine et al. (2014) and Tchuenche et al (2014) show that as the evolution of drug resistance grows, so does the number of infections in the population. However, these models do not consider the transmission of resistant infections - mosquitoes are either infected or susceptible only such that resistance only occurs from evolution within a treated host. More recently, Legros and Bonhoeffer (2016) modelled the resistance within-host, and used this model to determine the transmission rates in a simple compartmental (susceptible-infected) model. Unlike the other models in Table 1, there is not a separation of hosts infected with sensitive or resistant infections, since resistance is incorporated in the transmission rates, which depend on the within-host model of the density of gametocytes.

Generally, compartmental models which explicitly include a resistant class, do not include a partially-resistant class, although field evidence suggests that assuming only sensitive and fully-resistant gene classes is often invalid (Hastings et al. 2002). Resistance is a process, and thus better represented as a scale than a dichotomy. Tchuenche et al (2014), who do include partial resistance, do not include this class within the mosquito population, and thus ignoring the transmission of partially resistant infections. This is particularly relevant when considering the spread of resistance.

To summarise the overall findings of the compartmental models in Table 1, drug resistance increases in the population as treatment increases, and decreases as the period of infection decreases (drug efficacy increases). This is agreement with Mackinnon's (2005) summary on population genetics and epidemiological models. When the evolution of drug resistance is included, it is found to be a driving factor, but a comparison of this factor to the transmission probabilities of sensitive and resistant infections is currently missing. This omission has become more important as recent work interfaces within-host models with population models via these probabilities (Legros and Bonhoeffer, 2016 and Bushman et al., 2018).

This paper presents a novel compartmental model that includes the evolution of an infection within a treated host, such that a sensitive infection becomes a partially resistant infection, which becomes a fully resistant infection. This transference is defined by the 'replacement rate'. The replacement rate is a summary statistic that could be interpreted as an evolution rate which leads to the emergence of resistance. There has been a variety of 
approaches to model the emergence of resistance (Day et al., 2015, Day et al, 2016, Hastings, 2003, Hastings and Hodel, 2014, Hastings et al., 2002, Hastings and Watkins, 2005, MacKinnon, 2005, Read et al. 2011, Stepniewska and White, 2008, zur Weisch et al., 2011). In Section 4 we demonstrate how three different approaches can be combined with our model to interface within host models with population models.

Since 'replacement rate' is a summary statistic, it's definition is flexible to the question at hand, and thus the definition of partial resistance. For example, when treatment is a combination of two drugs - partial resistance may represent that the host has developed resistance to one drug, but not the second. Alternatively, resistance may require several mutations, as is the case with sulphadoxine pyrimethamine which has five important point mutations that have been found to be associated with resistance (Sarmah et al., 2017). These mutations occur incrementally, and thus less than five mutations can be considered as partial resistance. In fact, instead of one level of partial resistance, the model could be adapted to have four levels of partial resistance, one for each mutation. See Subsection 3.4 for further discussion about increasing the number of partially resistant classes.

In this model, the three different classes of infections are passed to mosquitoes such that mosquitoes can transmit partially resistant and fully resistant infections, where different classes of infections have different probabilities of being transmitted due to a cost of resistance. The key contribution of this paper is that we quantify the great importance of understanding the evolution of drug resistance - the replacement rate. Comparing this replacement rate to transmission properties, we show that controlling the emergence of drug resistance within a host is more effective than controlling the spread.

Our model compliments current research on resistance since it is this precise replacement rate that other research attempts to quantify, either by pharmacokinetic/pharmacodynamic modeling analysis (Hastings et al. 2002), theoretical modelling (Day and Read 2016), or within-host models (Bushman et al. 2016). The interface between this research and our model is discussed more in Section 4. Our model suggests that in areas of high transmission, the effect of the replacement rate is greater, so it is more important to minimise it by, for example, using drugs with a short half life (Hastings et al. 2002).

We do not include factors such as age structure, socio-economic factors, and migration since a malaria model that incorporates all factors and variables becomes an overwhelmingly complex system (Mandal et al. 2011). 
Table 1: The human and mosquito compartments used by previous malaria transmission
models which include a resistant population: Koella and Antia (2003), K, Esteva et al.
(2009), E, Chiyaka et al. (2009), C; Legros and Bonhoeffer (2011) (where immunity is
modelled within host - denoted by *); Tchuenche et al. (2011); Tumwiine et al. (2014);
and this paper, LP. All models include a susceptible population of humans and mosquitoes,

Table 1: The human and mosquito compartments used by previous malaria transmission
models which include a resistant population: Koella and Antia (2003), K, Esteva et al.
(2009), E, Chiyaka et al. (2009), C; Legros and Bonhoeffer (2011) (where immunity is
modelled within host - denoted by *); Tchuenche et al. (2011); Tumwiine et al. (2014);
and this paper, LP. All models include a susceptible population of humans and mosquitoes,

Table 1: The human and mosquito compartments used by previous malaria transmission
models which include a resistant population: Koella and Antia (2003), K, Esteva et al.
(2009), E, Chiyaka et al. (2009), C; Legros and Bonhoeffer (2011) (where immunity is
modelled within host - denoted by *); Tchuenche et al. (2011); Tumwiine et al. (2014);
and this paper, LP. All models include a susceptible population of humans and mosquitoes,

Table 1: The human and mosquito compartments used by previous malaria transmission
models which include a resistant population: Koella and Antia (2003), K, Esteva et al.
(2009), E, Chiyaka et al. (2009), C; Legros and Bonhoeffer (2011) (where immunity is
modelled within host - denoted by *); Tchuenche et al. (2011); Tumwiine et al. (2014);
and this paper, LP. All models include a susceptible population of humans and mosquitoes,

Table 1: The human and mosquito compartments used by previous malaria transmission
models which include a resistant population: Koella and Antia (2003), K, Esteva et al.
(2009), E, Chiyaka et al. (2009), C; Legros and Bonhoeffer (2011) (where immunity is
modelled within host - denoted by *); Tchuenche et al. (2011); Tumwiine et al. (2014);
and this paper, LP. All models include a susceptible population of humans and mosquitoes, omitted from the table for clarity.

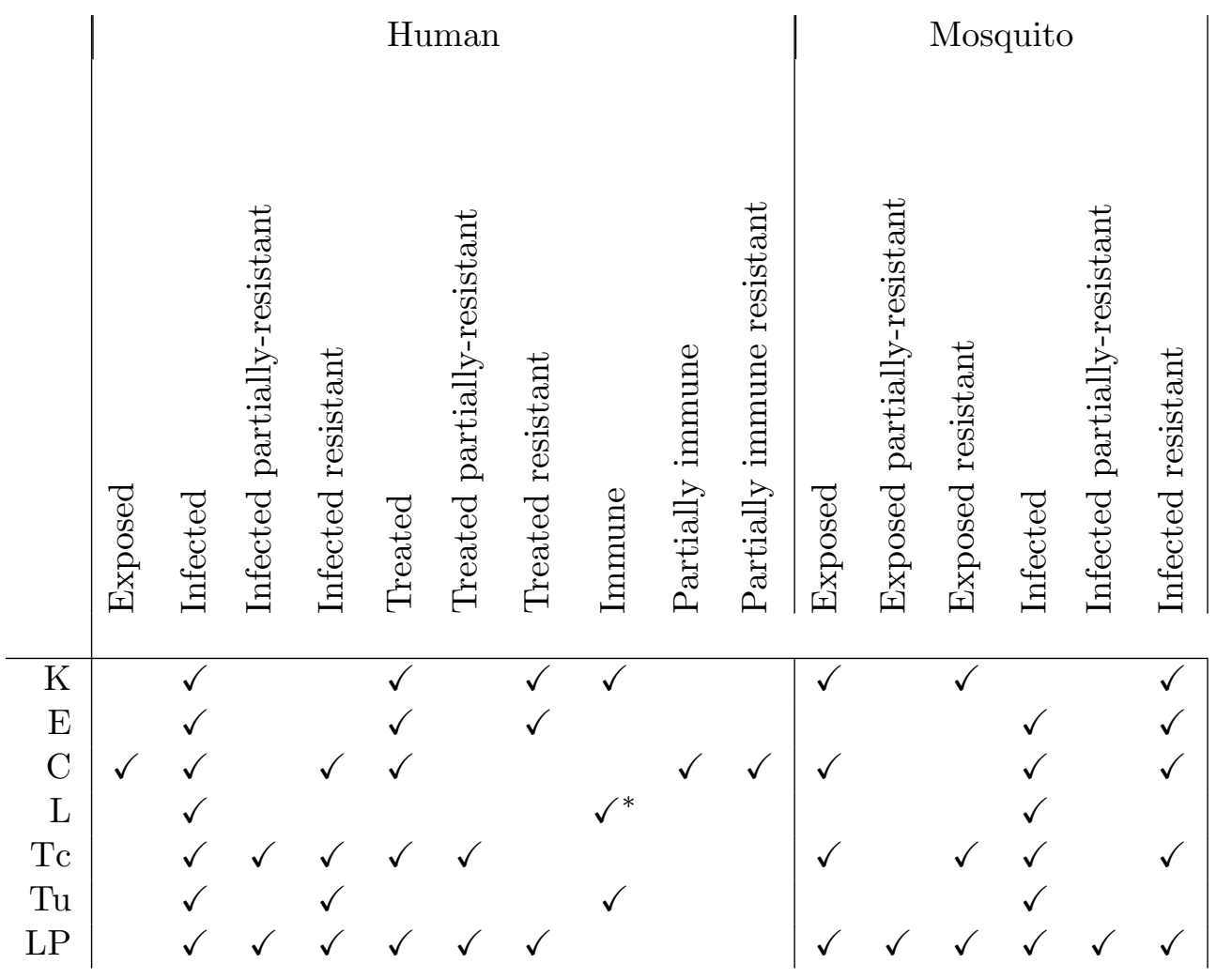

Moreover, our aim is to quantify the effect of treatment, and highlight what treatment and resistance variables are of most importance, so we include the minimum factors required. This is an introductory model that can act as the foundation for further studies which include multiple infections, and immunity. 
are either susceptible or infected. Infected mosquitoes bite susceptible humans who then become infected. Mosquitoes which bite infected humans become exposed (infected but not infectious), and after $\hat{\tau}$ time, become infectious if they have not already recovered. It is assumed that mosquito and human populations are constant. To model drug resistance, a treated human population is required, so we allow infections be to treated at a rate $r_{x}$, see Figure 1.

A novel aspect of this model is to follow three distinct infection classes: sensitive $j=\mathcal{S}$, partially-resistant $j=\mathcal{P}$, and fully-resistant $j=\mathcal{R}$ in both the human and mosquito population. Transference between the three classes, of the form $\mathcal{S} \rightarrow \mathcal{P} \rightarrow \mathcal{R}$, occurs in the treated population only, via a process we call 'replacement'. Replacement depends on factors such as the drug pressure, the mutation rate, and the de novo hazard. At a practical level these factors depend on inadequate dosage levels, poor compliance, combination therapy, and other implementation factors. Three different methods to quantify the replacement rate, $\phi$, are discussed in Section 4.

Resistance also occurs in the human population via mosquito transmission, which we consider separately since the resistance evolution is not directly affected by the transmission intensity (Hastings et al. 2005). Resistant infections may not be transmitted as easily, which is included in our model via the transmission probabilities $b_{j}$ and $c_{j}$. The probabilities that a bite leads to an infection in a human are related such that $b_{\mathcal{S}} \geq b_{\mathcal{P}} \geq b_{\mathcal{R}}$, and the probabilities that a bite leads to an infection in a mosquito are related such that $c_{\mathcal{S}} \geq c_{\mathcal{P}} \geq c_{\mathcal{P}}$. This allows the possibility that even when the cost of resistance is infinite, and so transmission of fully-resistant infections is zero, $b_{\mathcal{R}}=c_{\mathcal{P}}=0$, fully-resistant infections can persist due to the replacement of partially-resistant infections within treated hosts. Additionally, even when within-host resistance evolution has been removed, $\phi=0$, resistant infections may persist via transmission.

\subsection{Human population}

The total number of humans, which remains constant, is $N$, and is thus the sum of susceptible hosts $S$, infected hosts $I_{j}$ and treated hosts $T_{j}(j=$ $\mathcal{S}, \mathcal{P}, \mathcal{R})$

$$
N=S(t)+I(t)+T(t),
$$

where $I=I_{\mathcal{S}}+I_{\mathcal{P}}+I_{\mathcal{R}}$ and $T=T_{\mathcal{S}}+T_{\mathcal{P}}+T_{\mathcal{R}}$. The recovery and death rates for all untreated infections, and treated fully-resistant infections, are assumed 
to be the same, ( $r_{I}$ and $\alpha$ respectively). Perfect treatment is assumed so that the death rate for individuals with sensitive and partially-resistant treated infections is the same as the background death rate $\mu$. The recovery rates for sensitive and partially-resistant infections are $r_{T_{\mathcal{S}}}$ and $r_{T_{\mathcal{P}}}$. Comparing the recovery and death rates for the different classes: $r_{I}<r_{T_{\mathcal{P}}}<r_{T_{\mathcal{S}}}$ and $\mu<\alpha$.

\subsubsection{Infected human population, $I_{j}(t)$}

There are three classes of infected humans. For a given infection, the population is increased by the susceptible population which are bitten by a mosquito in class $j$, and decreased according to a recovery rate and background death rate,

$$
\frac{\mathrm{d} I_{j}}{\mathrm{~d} t}=a b_{j} m \frac{S}{N} \hat{I}_{j}-\left(r_{I}+r_{x}+\alpha\right) I_{j},
$$

where $a$ is the biting rate of mosquitoes, $m$ is the density of female mosquitoes, and $\hat{I}_{j}$ is the number of mosquitoes with a class $j$ infection. All variables and parameters are defined in Tables 2 and 3.

\subsubsection{Treated human population, $T_{j}(t)$}

Infected humans are treated at a rate $r_{x}$. Within the treated population, there is replacement $(\mathcal{S} \rightarrow \mathcal{P} \rightarrow \mathcal{R})$ due to growing resistance, via the replacement rate $\phi$,

$$
\begin{aligned}
\frac{\mathrm{d} T_{\mathcal{S}}}{\mathrm{d} t} & =r_{x} I_{\mathcal{S}}-\left(\phi+\mu+r_{T_{\mathcal{S}}}\right) T_{\mathcal{S}}, \\
\frac{\mathrm{d} T_{\mathcal{P}}}{\mathrm{d} t} & =r_{x} I_{\mathcal{P}}-\left(\phi+\mu+r_{T_{\mathcal{P}}}\right) T_{\mathcal{P}}+\phi T_{\mathcal{S}}, \\
\frac{\mathrm{d} T_{\mathcal{R}}}{\mathrm{d} t} & =r_{x} I_{\mathcal{R}}-\left(\alpha+r_{I}\right) T_{\mathcal{R}}+\phi T_{\mathcal{P}} .
\end{aligned}
$$

The replacement $\mathcal{S} \rightarrow \mathcal{P}$ is assumed to occur at the same rate as $\mathcal{P} \rightarrow \mathcal{R}$ for ease of analysis, but they could be different rates. Note that $I_{\mathcal{R}}$ and $T_{\mathcal{R}}$ are the same: fully-resistant infections are unaffected by treatment, either because treatment was not administered $I_{\mathcal{R}}$ or it is ineffective $T_{\mathcal{R}}$. However, using separate compartments allows us to monitor for infections that were initially partially-resistant $T_{\mathcal{R}}$ (establishment), and those that arise from mosquito transmission $I_{\mathcal{R}}$ (development). 
and we assume recovery rates and death rates do not vary due to infection. There are three classes of mosquitoes to correspond with the sensitive, partially-resistant, and fully-resistant infections.

\subsubsection{Exposed mosquito population, $E_{j}(t)$}

For a given infection, $j=\mathcal{S}, \mathcal{P}, \mathcal{R}$, the population is increased by the susceptible population which bite an infectious human in class $j$. At each time interval, a proportion leave the exposed population because the latency period $\hat{\tau}$ has expired, as well as the background death rate $\hat{\mu}$,

$$
\frac{\mathrm{d} \hat{E}_{j}}{\mathrm{~d} t}=a c_{j} \frac{I_{j}+T_{j}}{N} \hat{S}-a c_{j} \frac{I_{j}^{\prime}+T_{j}^{\prime}}{N^{\prime}} \hat{S}^{\prime} e^{-\hat{\mu} \hat{\tau}}-\hat{\mu} \hat{E}_{j},
$$

where $(\cdot)^{\prime}$ is $(\cdot)$ at time $t-\hat{\tau}$.

\subsubsection{Infected mosquito population, $\hat{I}_{j}(t)$}

The population is increased from the exposed population whose latency period has expired, and decreased according to the background death rate,

$$
\frac{\mathrm{d} \hat{I}_{j}}{\mathrm{~d} t}=a c_{j} \frac{I_{j}^{\prime}+T_{j}^{\prime}}{N^{\prime}} \hat{S}^{\prime} e^{-\hat{\mu} \hat{\tau}}-\hat{\mu} \hat{I}_{j} .
$$

\subsection{Example simulations}

The model is run for three years using the values in Table 3 , and initially no infected humans nor mosquitoes with partially-resistant nor fully resistant infections,

$$
I_{\mathcal{P}}=I_{\mathcal{R}}=\hat{E}_{\mathcal{P}}=\hat{E}_{\mathcal{R}}=\hat{I}_{\mathcal{P}}=\hat{I}_{\mathcal{R}}=0 .
$$

However, there are initially a small proportion of treated humans with the more resistant classes. The non-zero compartments at $t=0$ are

$S=99.4, I_{\mathcal{S}}=0.5, T_{\mathcal{S}}=T_{\mathcal{P}}=T_{\mathcal{R}}=0.099$, and $\hat{S}=80, \hat{E}_{\mathcal{S}}=\hat{I}_{\mathcal{S}}=10$ 


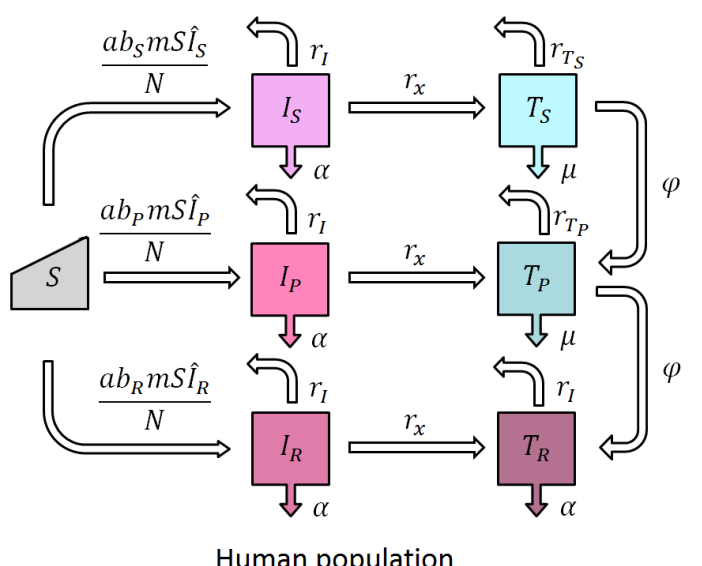

Human population

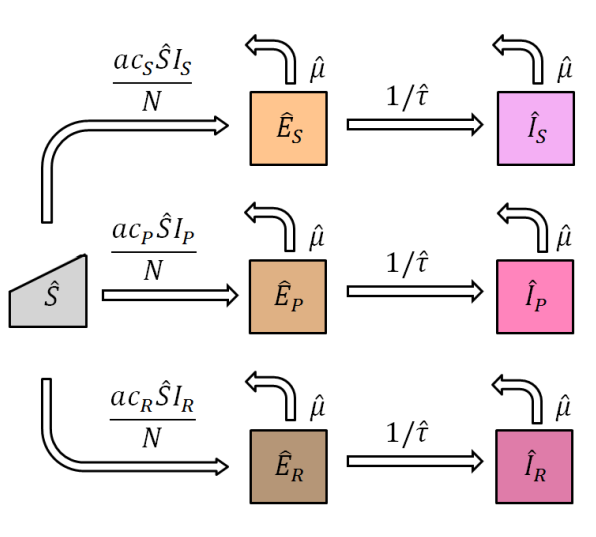

Mosquito population

Figure 1: Schematic diagram of our malaria model. Infections are either sensitive $j=\mathcal{S}$, a partially-resistant $j=\mathcal{P}$ or a fully-resistant $j=\mathcal{R}$. Susceptible mosquitoes become infected in relation to the proportion of infected humans. After a latency period of $\hat{\tau}$, mosquitoes become infectious and may infect susceptible humans. Infections in humans are treated at rate $r_{x}$. In the human population, each compartment has a down arrow to represent death, at either the background rate $\mu$, or the rate due to infection $\alpha(\mu<\alpha)$. The up arrows represent recovery $\left(r_{T_{\mathcal{S}}}<r_{T_{\mathcal{P}}}<r_{I}\right)$, where all recovered persons become susceptible again. For clarity, this return to susceptible is not explicitly shown. In the mosquito population, the up arrow represent death at rate $\hat{\mu}$, where death is replaced by new susceptible mosquitoes. 
Table 2: Model variables

\begin{tabular}{rc}
\hline Epidemiological compartments & Symbol \\
\hline Total number of humans & $N$ \\
Susceptible humans & $S$ \\
Humans with sensitive infection & $I_{\mathcal{S}}$ \\
Humans with partially-sensitive infection & $I_{\mathcal{P}}$ \\
Humans with fully-resistant infection & $I_{\mathcal{R}}$ \\
Total infected humans & $I$ \\
Treated humans with sensitive infection & $T_{\mathcal{S}}$ \\
Treated humans partially-sensitive infection & $T_{\mathcal{P}}$ \\
Treated humans fully-resistant infection & $T_{\mathcal{R}}$ \\
Total number of mosquitoes & $\hat{N}$ \\
Susceptible mosquitoes & $\hat{S}$ \\
Mosquitoes exposed to sensitive infection & $\hat{E_{\mathcal{S}}}$ \\
Mosquitoes exposed to partially-resistant infection & $\hat{E_{\mathcal{P}}}$ \\
Mosquitoes exposed to fully-resistant infection & $\hat{E_{\mathcal{R}}}$ \\
Total exposed mosquitoes & $\hat{E}$ \\
Mosquitoes infected with sensitive infection & $\hat{I_{\mathcal{S}}}$ \\
Total infected mosquitoes & $\hat{I_{\mathcal{I}}}$ \\
\hline Mosquitoes infected with partially-resistant infection & $\hat{I_{\mathcal{P}}}$ \\
Mosquitoes infected with fully-resistant infection & $\hat{I_{\mathcal{R}}}$ \\
\hline
\end{tabular}


Table 3: Parameter description and their default values. Unless indicated by a $*$, values are from Mandal et al. 2011. The values indicated by a $*$ are guesstimates.

\begin{tabular}{rcc}
\hline Parameter & Symbol & Value \\
\hline Natural death rate of humans & $\mu$ & $0.017 / 365$ day $^{-1}$ \\
Death rate of treated humans (assume perfect treatment) & $\mu$ & $0.017 / 365$ day $^{-1}$ \\
Death rate of not treated humans & $\alpha$ & ${ }^{*} 0.17 / 365$ day $^{-1}$ \\
Natural death rate of mosquitoes & $\hat{\mu}$ & 0.2 day $^{-1}$ \\
Latent period of mosquito & $\hat{\tau}$ & 11 days $^{-1}$ \\
Biting rate & $a$ & 0.25 day $^{-1}$ \\
Prob. that a bite transmits a sensitive infection to a human & $b_{\mathcal{S}}$ & 0.3 \\
Prob. that a bite transmits a partially-resistant infection to a human & $b_{\mathcal{P}}$ & 0.28 \\
Prob. that a bite transmits a fully-resistant infection to a human & $b_{\mathcal{R}}$ & 0.2 \\
Prob. that a bite transmits a sensitive infection to a mosquito & $c_{\mathcal{S}}$ & 0.5 \\
Prob. that a bite transmits a partially-resistant infection to a mosquito & $c_{\mathcal{P}}$ & 0.4 \\
Prob. that a bite transmits a fully-resistant infection to a mosquito & $c_{\mathcal{R}}$ & 0.3 \\
Ratio of female mosquitoes to humans & $m$ & 28 \\
Rate that infected humans receive treatment & $r_{x}$ & 0.03 day $^{-1}$ \\
Average recovery rate of untreated infections & $r_{I}$ & 0.02 day $^{-1}$ \\
Average recovery rate of treated, sensitive infections & $r_{T_{\mathcal{S}}}$ & ${ }^{*} 0.06$ day $^{-1}$ \\
Average recovery rate of treated, partially-resistant infections & $r_{T_{\mathcal{P}}}$ & ${ }^{*} 0.04$ day $^{-1}$ \\
Replacement rate & $\phi$ & ${ }^{*} 1 / 110$ day $^{-1}$ \\
\hline
\end{tabular}

Despite a very low initial presence of resistance, the proportion of resistant infections grows rapidly, but then it appears that an endemic equilibrium is reached, see Figure 2a. Whereas when there is no cost of resistance, such that $b_{\mathcal{S}}=b_{\mathcal{P}}=b_{\mathcal{R}}$ and $c_{\mathcal{S}}=c_{\mathcal{P}}=c_{\mathcal{R}}$ (Figure $2 \mathrm{~b}$ ), the infected proportion continues to increase. The specific requirements for an endemic equilibrium, and the effect of the transmission probabilities, is discussed further in Section 3.2.

As previously mentioned, even when $b_{\mathcal{R}}=c_{\mathcal{R}}=0$, fully-resistant infections persist due to the replacement of partially-resistant infections within treated hosts, see Figure 2c. This figure also has the rate of replacement set to zero after one year. Together with Figure 2d, this shows that resistance persists in a population once once the possibility of resistance developing within a host is removed.

\section{Results}

Having established the model, and discussed some examples, we present some analyses to track the emergence and spread of resistance. In Section 3.1, the number of secondary infections arising from a single infection is calcu- 


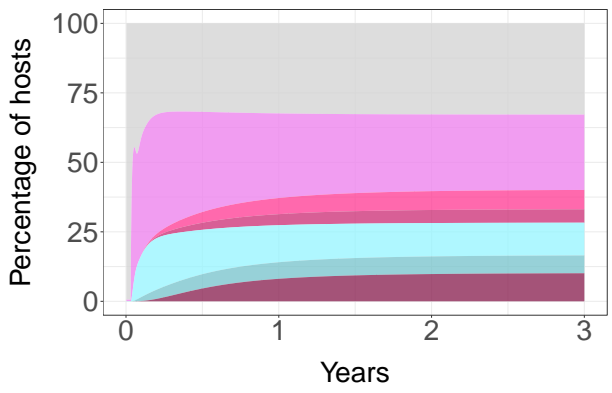

(a) All parameters as in Table $3\left(b_{\mathcal{S}}=\right.$ $0.3, b_{\mathcal{P}}=0.28, b_{\mathcal{R}}=0.2$ and $c_{\mathcal{S}}=$ $\left.0.5, c_{\mathcal{P}}=0.4, c_{\mathcal{P}}=0.3\right)$.

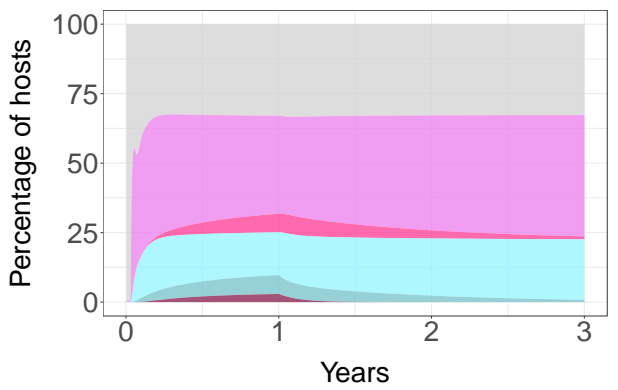

(c) Fully resistance infections cannot be transmitted, $b_{\mathcal{R}}=c_{\mathcal{R}}=0$, and withinhost resistance evolution removed after one year, $\phi=0$ for $t>365$.

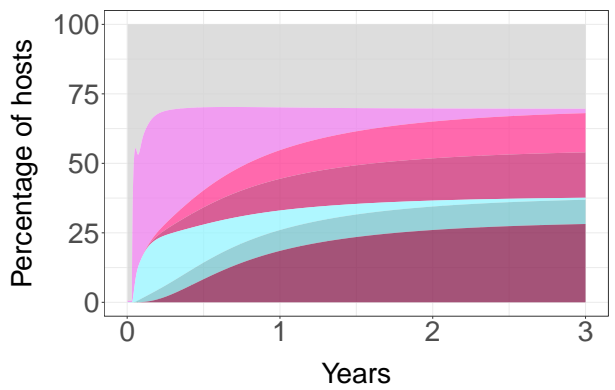

(b) All infections equally likely to be transmitted, $b_{\mathcal{S}}=b_{\mathcal{P}}=b_{\mathcal{R}}=0.3$ and $c_{\mathcal{S}}=c_{\mathcal{P}}=c_{\mathcal{P}}=0.5$.

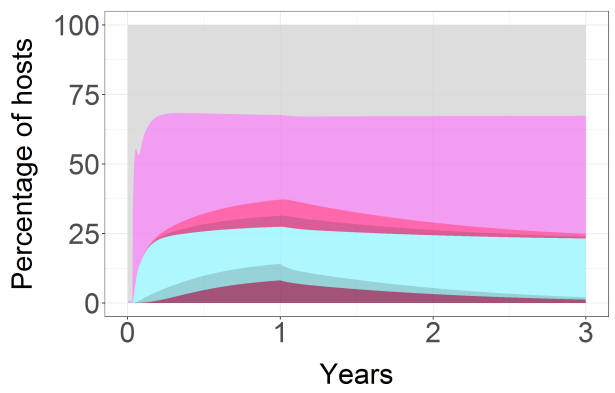

(d) Within-host resistance evolution removed after one year, $\phi=0$ for $t>365$.

Figure 2

Example simulations to show the effect of varying transmission probabilities and within-host evolution. All parameters are as in Table 3, unless otherwise stated. The colours correspond to the compartment colours in

Figure 1: Pink compartments are the infected populations, without treatment, and the blue compartments are the treated population. Except the darkest pink, which is the treated population with the fully resistant infection (this compartment is equivalent to the fully-resistant infected population without treatment, second darkest pink). The darker tone correspond to more resistant infections.

lated. By keeping the three classes of infections separate, we focus on the number of resistant infections that arise from a sensitive infection. The affect of the different treatment variables is discussed, and the importance of the 
replacement rate $\phi$ is quantified. Then we discuss the requirements for an endemic equilibrium.

The importance of $\phi$ is verified for two model adaptations, which would make the model more realistic. Firstly, in Subsection 3.3, we show that the results remain the same when an asymptomatic human population is included. Secondly, in Subsection 3.4, we show that as more levels of resistance evolution are included, the replacement rate $\phi$ actually becomes more important.

\subsection{Reproductive numbers}

As resistance grows, so does the total number of infected individuals. The reproductive number is a measure of the number of secondary, infectious, infections expected after one new infection. The number of infections of any class, arising from a single infection of any class, is denoted by $R_{\mathcal{S}} \mathcal{P} R \rightarrow \mathcal{S} \mathcal{P} \mathcal{R}$ and is the sum of

$$
\begin{aligned}
& \left.\begin{array}{r}
R_{I_{\mathcal{S}} \rightarrow \hat{I}_{\mathcal{S}}} R_{\hat{I}_{\mathcal{S}} \rightarrow I_{\mathcal{S}}} \\
R_{I_{\mathcal{S}} \rightarrow T_{\mathcal{S}} \rightarrow \hat{I}_{\mathcal{S}}} R_{\hat{I}_{\mathcal{S}} \rightarrow I_{\mathcal{S}}}
\end{array}\right\} R_{\mathcal{S} \rightarrow \mathcal{S}}, \\
& \left.R_{R_{I_{\mathcal{P}} \rightarrow T_{\mathcal{P}} \rightarrow \hat{I}_{\mathcal{P}}} R_{\hat{I}_{\mathcal{P}} \rightarrow I_{\mathcal{P}}}} \begin{array}{l}
R_{I_{\mathcal{P}} \rightarrow \hat{I}_{\mathcal{P}}} R_{\hat{I}_{\mathcal{P}} \rightarrow I_{\mathcal{P}}} \\
R_{T_{\mathcal{P}}}
\end{array}\right\} R_{\mathcal{P} \rightarrow \mathcal{P}}, \\
& \left.\begin{array}{r}
R_{I_{\mathcal{R}} \rightarrow \hat{I}_{\mathcal{R}}} R_{\hat{I}_{\mathcal{R}} \rightarrow I_{\mathcal{R}}} \\
R_{I_{\mathcal{R}} \rightarrow T_{\mathcal{R}} \rightarrow \hat{I}_{\mathcal{R}}} R_{\hat{I}_{\mathcal{R}} \rightarrow I_{\mathcal{R}}}
\end{array}\right\} R_{\mathcal{R} \rightarrow \mathcal{R}}, \\
& \left.R_{I_{\mathcal{S}} \rightarrow T_{\mathcal{S}} \rightarrow T_{\mathcal{P}} \rightarrow \hat{I}_{\mathcal{P}}} R_{\hat{I}_{\mathcal{P}} \rightarrow I_{\mathcal{P}}}\right\} R_{\mathcal{S} \rightarrow \mathcal{P}}, \\
& \left.R_{I_{\mathcal{P}} \rightarrow T_{\mathcal{P}} \rightarrow T_{\mathcal{R}} \rightarrow \hat{I}_{\mathcal{R}}} R_{\hat{I}_{\mathcal{R}} \rightarrow I_{\mathcal{R}}}\right\} R_{\mathcal{P} \rightarrow \mathcal{R}}, \\
& \left.R_{I_{\mathcal{S}} \rightarrow T_{\mathcal{S}} \rightarrow T_{\mathcal{P}} \rightarrow T_{\mathcal{R}} \rightarrow \hat{I}_{\mathcal{R}}} R_{\hat{I}_{\mathcal{R}} \rightarrow I_{\mathcal{R}}}\right\} R_{\mathcal{S} \rightarrow \mathcal{R}},
\end{aligned}
$$

where the subscripts indicate the movement of the initial infection through the different compartments. For example, infection of class $j$ in a mosquito passed to a human is $R_{\hat{I}_{j} \rightarrow I_{j}}$. The reproductive numbers (12)-(14) relate to resistance emerging, with (14) being of particular interest as it relates to the number of fully resistant infections arising from a single sensitive infection. Once fully resistant infections are established, the reproductive number (11) relates to the fully resistant infection spreading. From the model, (9)-(14) are defined as,

$$
R_{\mathcal{S} \rightarrow \mathcal{S}}=\frac{a^{2} b_{\mathcal{S}} c_{\mathcal{S}} m}{\hat{\mu}}\left[\frac{r_{T_{\mathcal{S}}}+r_{x}+\phi+\mu}{\left(r_{I}+r_{x}+\alpha\right)\left(r_{T_{\mathcal{S}}}+\phi+\mu\right)}\right] e^{-\hat{\mu} \hat{\tau}}
$$




$$
\begin{aligned}
& R_{\mathcal{P} \rightarrow \mathcal{P}}=\frac{a^{2} b_{\mathcal{P}} c_{\mathcal{P}} m}{\hat{\mu}}\left[\frac{r_{T_{\mathcal{P}}}+r_{x}+\phi+\mu}{\left(r_{I}+r_{x}+\alpha\right)\left(r_{T_{\mathcal{P}}}+\phi+\mu\right)}\right] e^{-\hat{\mu} \hat{\tau}} \\
& R_{\mathcal{R} \rightarrow \mathcal{R}}=\frac{a^{2} b_{\mathcal{R}} c_{\mathcal{P}} m}{\hat{\mu}}\left[\frac{1}{r_{I}+\alpha}\right] e^{-\hat{\mu} \hat{\tau}}, \\
& R_{\mathcal{S} \rightarrow \mathcal{P}}=\frac{a^{2} b_{\mathcal{P}} c_{\mathcal{P}} m}{\hat{\mu}}\left[\frac{r_{x} \phi}{\left(r_{I}+r_{x}+\alpha\right)\left(r_{T_{\mathcal{S}}}+\phi+\mu\right)\left(r_{T_{\mathcal{P}}}+\phi+\mu\right)}\right] e^{-\hat{\mu} \hat{\tau}}, \\
& R_{\mathcal{P} \rightarrow \mathcal{R}}=\frac{a^{2} b_{\mathcal{R}} c_{\mathcal{P}} m}{\hat{\mu}}\left[\frac{r_{x} \phi}{\left(r_{I}+r_{x}+\alpha\right)\left(r_{T_{\mathcal{P}}}+\phi+\mu\right)\left(r_{I}+\alpha\right)}\right] e^{-\hat{\mu} \hat{\tau}}, \\
& R_{\mathcal{S} \rightarrow \mathcal{R}}=\frac{a^{2} b_{\mathcal{R}} c_{\mathcal{P}} m}{\hat{\mu}}\left[\frac{r_{x} \phi^{2}}{\left(r_{I}+r_{x}+\alpha\right)\left(r_{T_{\mathcal{S}}}+\phi+\mu\right)\left(r_{T_{\mathcal{P}}}+\phi+\mu\right)\left(r_{I}+\alpha\right)}\right] e t \hat{\boldsymbol{2} \hat{0} .)}
\end{aligned}
$$

The derivation of (15)-(20) is provided in Appendix A. The terms outside the square brackets relate to the reproductive number of the delay RossMcDonald model,

$$
R_{\mathcal{S}}=\frac{a^{2} b c m e^{-\hat{\mu} \hat{\tau}}}{\hat{\mu}}
$$

(Ruan et al. 2009). That is, reduction in transmission is most strongly affected by the exponent terms: the death rate of mosquitoes $\hat{\mu}$, and the latency time period $\hat{\tau}$; the biting rate $a$ has a stronger affect than the transmission probabilities $b, c$ (here $b_{j}, c_{j}$ ) and the mosquito density $m$. These known affects have more impact for the reproductive numbers (15)-(20) which have a larger term inside the square brackets. This is especially true for the variables to the left of the square brackets, which relate to transmission rates, because the terms in the square brackets are of the same order as the transmission terms, whereas the latency period and mosquito death rate are exponents.

We now investigate when the reproductive numbers relating to resistance emerging (18)-(20) are large for varying parameters, and thus informing when the transmission rates and latency period have a stronger effect on the spread of resistance.

Let us assume that the recovery rate from infection $r_{I}$, the death rate from infection $\alpha$ and background death rate $\mu$ are fixed. Therefore, the reproductive numbers (15)-(20) only vary by the treatment variables:

- the recovery rates for sensitive and partially-resistant infections which are being treated, $r_{T_{\mathcal{S}}}, r_{T_{\mathcal{P}}}$. It is assumed that these rates range between 2 and 10 times larger than the recovery rate of non-treated individuals $r_{I}$, 
- the rate of infections being replaced by more resistant infections $\phi \in$ $[0,1]$

- the treatment rate $r_{x} \in(0,1]$;

and the cost of resistance, represented by the transmission probabilities $b_{j}$ and $c_{j}$. Overall, the number of infections can be reduced by increasing the treatment rate $r_{x}$ and increasing the recovery rates, $r_{T_{\mathcal{S}}}$ and $r_{T_{\mathcal{P}}}$. However, as the replacement rate $\phi$ increases, so does the total number of infections, see Figure 4a.

Previous studies confirm that increasing the rate of treatment increases the rate of resistance spread (Koella and Antia 2003, Esteva et al. 2009, Chiyaka et al. 2009); and increasing the cost of resistance reduces the spread of resistance (Koella and Antia 2003, Esteva et al. 2009). However, like Tchuenche et al (2011) and Tumwiine et al. (2014) who include resistance growth, we show that the replacement rate has a much stronger affect, see Figure 3. Moreover, by including partial resistance in the mosquito population, we can separate resistant infections that are transmitted and those that develop within the host.

When the replacement rate is high, there are a lot of secondary fullyresistant infections arising from a single sensitive infection, $R_{\mathcal{S} \rightarrow \mathcal{R}}$. This is because the development of fully-resistant infections from sensitive infections, $R_{\mathcal{S} \rightarrow \mathcal{R}}$, is affected by the replacement rate twice, hence equation (20) is $O\left(\phi^{2}\right)$, which is the same order as the biting rate $a$. Therefore, in this model, the replacement rate has an equal affect as the biting rate. Since the replacement rate $\phi$ and the transmission variables $a, b_{j}, c_{j}$ and $m$ are of similar order, in areas of high transmission, reducing the replacement rate has a strong effect. Moreover, this is actually more effective than reducing the treatment rate $r_{x}$, or increasing the cost of resistance $b_{j}, c_{j}$, at mitigating resistance spread. The limited effect of transmission rates $b_{j}$ and $c_{j}$ is in agreement with Gandon et al. (2001, 2003), where they showed that vaccines limiting transmission have little effect on evolution.

Nonetheless, one must be careful when interpreting the specifics of this model. For example, treatment rate per day is likely to be considerably larger than the replacement rate per day. Under this model, there is reason to believe that under certain conditions, the treatment rate may actually create more resistance emergence than the replacement rate (for example, the treatment rate is $r_{x}=0.9$ and the replacement rate is $\phi=0.01$, see Figure 4b). However, as we discuss later in Section 3.4, only including one step to 
resistance is perhaps still too coarse a lens, which would make this example meaningless. Instead, when interpreting the resistance emerging reproductive number $R_{\mathcal{S} \rightarrow \mathcal{R}}$, consider the overall result that whilst the influence of the replacement rate is compounded, the treatment rate and transmission rates remain unchanged.

All resistance emerging reproductive numbers, $R_{\mathcal{S} \rightarrow \mathcal{P}}, R_{\mathcal{P} \rightarrow \mathcal{R}}, R_{\mathcal{S} \rightarrow \mathcal{R}},(15)$ (20) are inversely related to the treatment rates $r_{T_{\mathcal{S}}}$ and $r_{T_{\mathcal{P}}}$. Therefore, improved recovery rates not only reduce the overall number of infections, but it is especially beneficial for reducing the emergence of drug resistance. Note that $R_{\mathcal{S} \rightarrow \mathcal{P}}, R_{\mathcal{P} \rightarrow \mathcal{R}}, R_{\mathcal{S} \rightarrow \mathcal{R}},(15)-(20)$ depend on the treatment rate of partially-resistant infections $r_{T_{\mathcal{P}}}$, whereas the treatment rate of sensitive infections $r_{T_{\mathcal{S}}}$ affects $R_{\mathcal{S} \rightarrow \mathcal{P}}$ and $R_{\mathcal{P} \rightarrow \mathcal{R}}$ only. Therefore the recovery rate of partially-resistant infections is of more importance at mitigating the spread of resistance. In fact, the recovery rate of partially-resistant infections $r_{T_{\mathcal{P}}}$ has a stronger affect than the treatment rate $r_{x}$, see Figure 4a.

\subsection{Equilibrium}

When considering the disease free equilibrium, we consider the case of a constant human population. Without treatment, the system reduces to the delay Ross-McDonald model, and thus the disease free equilibrium is trivial. The endemic equilibrium is

$$
\frac{I^{*}}{N}=\frac{R_{\mathcal{S}}-1}{R_{\mathcal{S}}+a c e^{-\hat{\mu} \hat{\tau}} / \hat{u}},
$$

where $R_{\mathcal{S}}$ is as in (21), from Ruan et al. 2009. For the full model presented here, with treatment and resistance, the disease free equilibrium is when

$$
\begin{aligned}
I_{j}^{*} & =\frac{\beta_{j}}{r_{I}+r_{x}+\alpha}, \\
T_{\mathcal{S}}^{*} & =\frac{r_{x} \beta_{\mathcal{S}}}{\left(r_{I}+r_{x}+\alpha\right)\left(r_{T_{\mathcal{S}}}+\phi+\mu\right)}, \\
T_{\mathcal{P}}^{*} & =\frac{r_{x}\left[\phi \beta_{\mathcal{S}}+\left(r_{T_{\mathcal{S}}}+\phi+\mu\right) \beta_{\mathcal{P}}\right]}{\left(r_{I}+r_{x}+\alpha\right)\left(r_{T_{\mathcal{S}}}+\phi+\mu\right)\left(r_{T_{\mathcal{P}}}+\phi+\mu\right)}, \\
T_{\mathcal{R}}^{*} & =\frac{r_{x}\left[\phi^{2} \beta_{\mathcal{S}}+\phi\left(r_{T_{\mathcal{S}}}+\phi+\mu\right) \beta_{\mathcal{P}}+\left(r_{T_{\mathcal{S}}}+\phi+\mu\right)\left(r_{T_{\mathcal{P}}}+\phi+\mu\right) \beta_{\mathcal{R}}\right]}{\left(r_{I}+r_{x}+\alpha\right)\left(r_{T_{\mathcal{S}}}+\phi+\mu\right)\left(r_{T_{\mathcal{P}}}+\phi+\mu\right)\left(r_{I}+\alpha\right)},
\end{aligned}
$$

where $\beta_{j}=a b_{j} m\left(S^{*} / N\right) \hat{I}_{j}^{*}$. As expected, when $\beta_{\mathcal{S}}=\beta_{\mathcal{P}}=\beta_{\mathcal{R}}=0$, the equilibrium is the disease free equilibrium. These conditions are not met in the examples in Figure 2, but it is clear that the difference is negligible. 

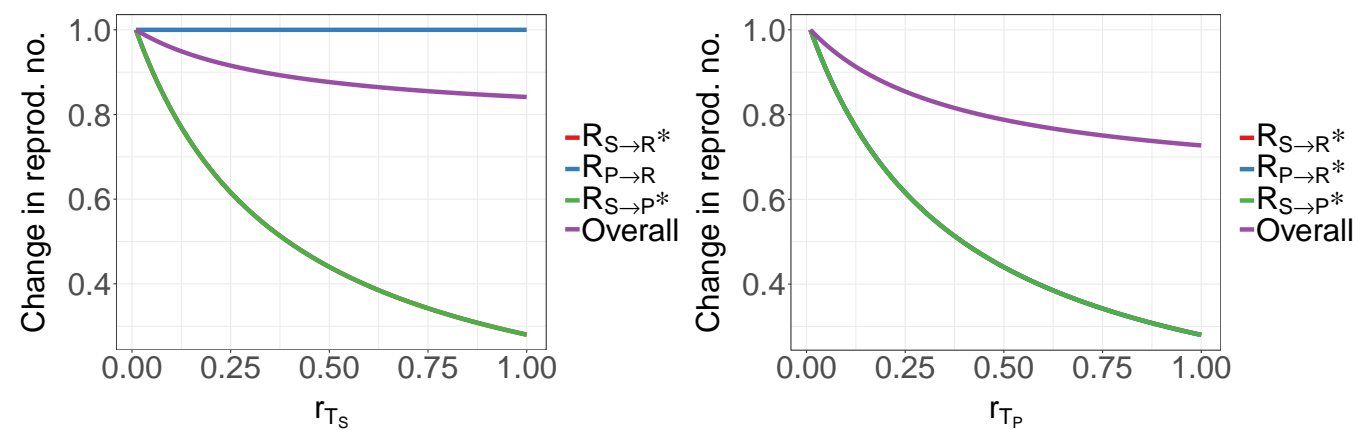

(a) The recovery rate of treated humans in(b) The recovery rate of treated humans in the sensitive class, $r_{T_{\mathcal{S}}}$. the partially-resistant class, $r_{T_{\mathcal{P}}}$.

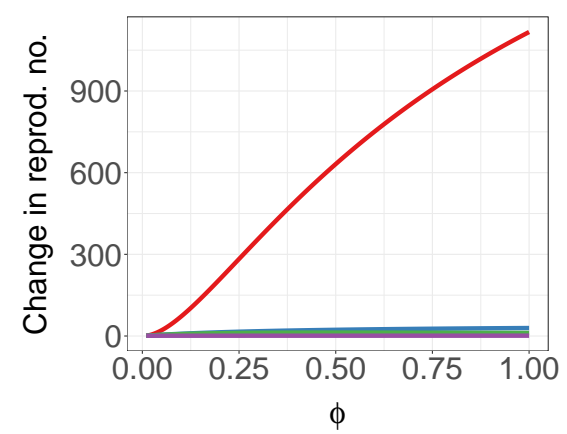

(c) The replacement rate, $\phi$.

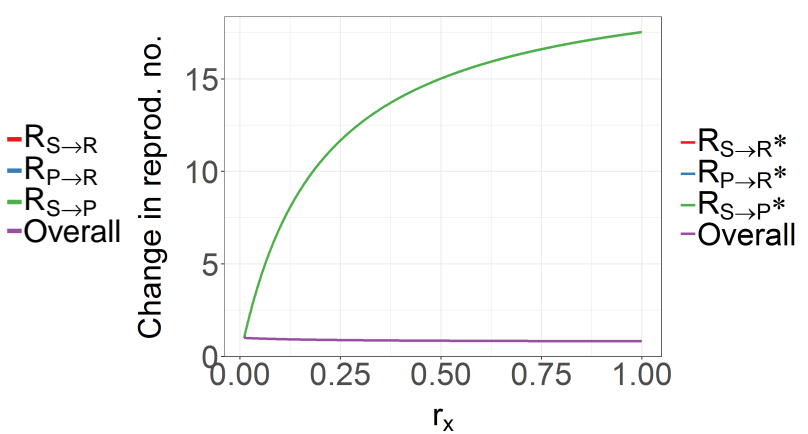

(d) The treatment rate, $r_{x}$.

Figure 3

The change in the resistance emerging reproductive numbers $R_{\mathcal{S} \rightarrow \mathcal{P}}, R_{\mathcal{P} \rightarrow \mathcal{R}}$

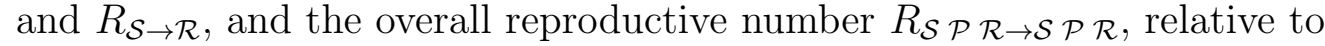
the recovery rate of the treated population $r_{T}$, the treatment rate $r_{x}$, and the replacement rate $\phi$. The ${ }^{*}$ in the legend indicates the lines are the same.

We now discuss the conditions required for an equilibrium where only the sensitive, and partially-resistant infections are present,

$$
I_{\mathcal{R}}=T_{\mathcal{R}}=\hat{I}_{\mathcal{R}}=0
$$

If fully-resistant infections are absent from $I_{\mathcal{R}}$ and $\hat{I}_{\mathcal{R}}$, they can only enter the population via $T_{\mathcal{P}}$. Therefore the equilibrium (22) will only remain stable if there is no movement from $T_{\mathcal{P}}$ to $T_{\mathcal{R}}$. From (19), and assuming that the death and recovery rates are non-zero, $\alpha, r_{I}>0$, it is clear that movement 
from $T_{\mathcal{P}}$ to $T_{\mathcal{R}}$ is only prevented if the treatment or replacement rate is zero, $r_{x}=0$ or $\phi=0$. Similarly, the conditions for sensitive only infections is only stable under the same conditions. Moreover, even when the cost of resistance prevents transmission of the fully-resistant infection, this class of infection persists unless $r_{x}=0$ or $\phi=0$ due to resistance developing within a treated host.

\subsection{Including an asymptomatic population}

An infection in a human may be asymptomatic $A_{j}$, such that infections in humans could transfer $S \leftrightarrow A_{j} \leftrightarrow I_{j} \rightarrow T_{j}$ (as well as $S \leftrightarrow I_{j} \rightarrow T_{j}$ as before), where $A_{j}$ represents asymptomatic infections. We now discuss how the reproductive numbers (15)-(20) change with this added feature.

With these new compartments, the recovery rate of infections (untreated or fully-resistant) $r_{I}$, is the sum of the recovery rates from infected to susceptible $r_{I S}$ and from infected to asymptomatic $r_{I A}$. Similarly, the recovery rates from treated infections $r_{T_{j}}, j=\mathcal{S}, \mathcal{P}$, is the sum of the recovery rates from treated to susceptible $r_{T_{j} S}$ and from treated to asymptomatic $r_{T_{j} A}$. This change does not alter the reproductive numbers (15)-(20). However, more significantly, an asymptotic population adds an exponential term $e^{-\left(r_{A}+\mu\right) \tau}$, where $r_{A}$ is the recovery rate of asymptotic infections, and $\tau$ is the asymptotic period. This highlights results consistent with previous models - the period of time that humans are infectious is key factor of transmission dynamics (Chiyaka et al. 2009).

\subsection{Resistance as a scale}

Consider a model for a single infection without partial-resistance, such that fully-resistant infections replace sensitive infections directly. In this circumstance the number of secondary fully-resistant infections arising from a single sensitive infection, $R_{\mathcal{S} \rightarrow \mathcal{R}}$, would be $O\left(b_{\mathcal{R}}\right), O\left(c_{\mathcal{P}}\right), O\left(r_{x}\right)$, and $O(\phi)$, not $O\left(\phi^{2}\right)$ as in this model. This would have led to the conclusion that transmission probabilities, treatment rate, and replacement rate are equally important. Alternatively, consider a single infection with $n$ steps towards full resistance, where each step is equally likely. Then the number of secondary fully-resistant infections arising from one sensitive infection $R_{\mathcal{S} \rightarrow \mathcal{R}}$, would be $O\left(b_{\mathcal{R}}\right), O\left(c_{\mathcal{P}}\right), O\left(r_{x}\right)$, as before, but now $O\left(\phi^{n}\right)$. Therefore, because infections evolve through different stages before becoming fully-resistant, controlling this evolution is incredibly important, and much more important than transmission probabilities and treatment rate. By modelling resistance 
emergence as a scale, and not a sensitive-resistant dichotomy, the potential of combination therapies to disrupt emergence comes into focus.

To demonstrate, suppose that a sensitive infection evolves resistance to a drug at rate $\phi_{A}$, and develops resistance to a partner drug at rate $\phi_{B}$. From our analysis we observe that the number of fully resistant infections to result from a single sensitive infection, $R_{\mathcal{S} \rightarrow \mathcal{R}}$, would be $O\left(\phi_{A} \phi_{B}\right)$. (Both rates relate to within-host evolution, so the conclusion that within-host dynamics is the driver of resistance still holds.) In this form it is clear that eliminating one step $\left(\phi_{A}=0\right.$ or $\left.\phi_{B}=0\right)$ prevents full resistance developing.

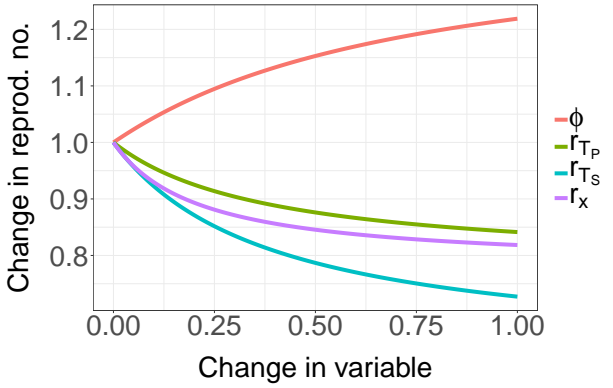

(a) $R_{\mathcal{S}, \mathcal{P}, \mathcal{R} \rightarrow \mathcal{S}, \mathcal{P}, \mathcal{R}}$

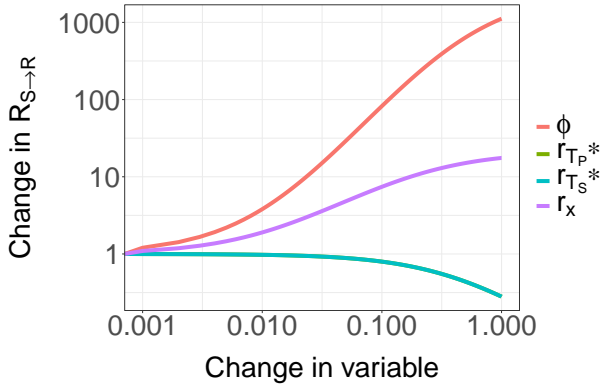

(b) $R_{\mathcal{S} \rightarrow \mathcal{R}}(\log )$

Figure 4

The change in the overall reproductive number and the resistance emerging reproductive number, relative to changes in the treatment variables. The ${ }^{*}$

in the legend indicates the lines are the same. Note that as the model becomes more realistic so as to incorporate additional levels of resistance, the effect of the recovery rates, $r_{T_{\mathcal{S}}}, r_{T_{\mathcal{P}}}$, and treatment rate, $r_{x}$, remain the same, but the effect of the replacement rate $\phi$ (the red line) increases dramatically.

\section{Parameterising the replacement rate $\phi$}

Having established that the replacement $\phi$ is the most important treatment variable, we discuss three different methods to determine an approximate value. This value is bounded by 0 , meaning no resistance evolution, and 1 , meaning instant transference from sensitive to resistant.

\subsection{The selection window}

When a treatment is first administered a patient is protected from partiallyresistant and sensitive infections. Once the drug concentration is below a 
certain value, the resistant genotypes are no longer inhibited by the drug and spreads to replace the original, sensitive infection. This time period is referred is referred to as the selection window (Kay and Hastings 2015, Hastings, Watkins and White 2002). Let us assume that during this selection window, sensitive parasites are 'replaced' by partially-resistant parasites, and thus $\phi$ is connected to the selection window.

Kay and Hastings (2015) use the selection window to calculate the probability, as a function of time, of parasites successfully surviving residual drug levels. They show that artmether-lumefantrine and artesunate-mefloquine kept the probability of successful emergence (our 'replacement') below $10 \%$ for 10 to 20 days post-treatment. This corresponds to $0.0055 \leq \phi \leq 0.011$. Whereas resistance is more likely to occur with DHA piperaquine, which kept the probability of successful emergence ('replacement') below $40 \%$ for 10 days post-treatment, $\phi=0.05$. We use a default value of $\phi=1 / 110=0.0091$ (see Table 3), which lies in the range of a combined artesunate treatment.

\subsection{The probability of resistance}

Day and Read (2016) calculate the probability of resistance, dependent on the drug concentration $c$. This corresponds to $\phi$ in our model such that

$$
\phi=1-e^{-H(c)},
$$

where $H(c)$ is the sum of the de novo hazard and the standing hazard. The de novo hazard depends on the rate which resistant mutations appear after the start of the treatment, and the probability of escape of any such mutant. The standing hazard is the hazard due to a standing population of resistant microbes that are already present at the start of treatment. The full equation for $H(c)$ is provided in Appendix B. Unlike Hastings, Watkins and White (2002), which focuses on the effect of drug pressure only, Day and Read (2016) find that sometimes moderate treatment is preferred. Equation (23) provides useful insight, but parameterising it remains an open challenge.

\subsection{Within-host modelling}

Lastly, one could model the dynamics within-host (Bushman et al. 2016), and interface the two models, as in Legros and Bonhoeffer (2016). In Legros and Bonhoeffer (2016), the transmission probability $b$, depends on the number of gametocytes, which is determined by a within-host model. The results 
from this paper indicate that it would be more important to include the replacement rate. This could be done by considering the erythrocytes infected with the sensitive clone, $Y_{\mathcal{R}}$, such that

$$
\phi=\epsilon\left(1-\mu_{y}\right) \mu_{m} Y_{\mathcal{R}}
$$

where $\epsilon \in[0,1]$ is the treatment efficacy, and $\mu_{y}$ is the death rate of infected erythrocytes, which are both included in the original Legros and Bonhoeffer (2016) model. The new variable $\mu_{m} \in[0,1]$ relates to the proportion of erythrocytes infected with the sensitive clone which evolve to become infected with the resistant clone. Replacement rate (24) allows a transference from sensitive to resistant erythrocytes that increases as the treatment efficacy increases, whilst still allowing for reduction in erythrocytes due to death. Of note, as the number of erythrocytes infected with the sensitive clone $Y_{\mathcal{R}}$ changes over time, so does the replacement rate $\phi$.

\section{Discussion}

Generally, previous models which monitor the spread of resistance have found that reducing the proportion of people treated is one of the most reliable ways to reduce resistance, which is clearly an undesirable strategy both for control and elimination. Our model agrees with this finding, but more encouragingly and realistically, reducing the replacement rate has a stronger effect at reducing resistance spread. Models which include the evolution of drug resistance show that it is important, but omit mosquitoes transmitting varying infections, so a comparison to the transmission probabilities is missing.

In fact, for a model that considers one partially-resistant class only, the effectiveness of this control strategy is directly comparable to the conclusions from the original Ross-MacDonald model which found that reducing the biting rate of mosquitoes is more effective than reducing density of mosquitoes. However, when one considers that resistance is a continuous scale, the evolution within-host is the most important factor, which emphasises the potential of combination therapies to disrupt emergence.

The replacement rate $\phi$ is not specific to a given drug, but instead it is a measure which can be influenced by implementation procedures, such as pharmacokinetics, pharmacodynamics, poor adherence or combination therapy. The parameterisation examples provided in Section 4 could be consid- 
ered as a single factor of a much more complex system. Whilst reasonably parameterising this more complex system may be overreaching, understanding the various factors should still be the focus of policy decisions. For example, when administering combination therapy, it may be challenging to understanding the different rates of resistance to individual drugs, but because it is understood that combination therapy lowers the overall replacement rate, it should be the preferred treatment strategy. This focus on keeping evolution low by treatment administration protocol is also discussed by Bell and MacLean (2016), who present an evo-epidemiological model of antibiotic resistance. Their work predicts that it should be possible for any antibiotic to be effectively evolution-proof, as long as the antibiotic is administered in a way that prevents the epidemic spread of resistant lineages.

\section{Conclusion}

As resistance spreads, treatment becomes ineffective. To understand drivers of resistance we developed a compartmental model that includes partial resistance and full resistance, and we then quantified the number of resistant infections that arise from a single sensitive infection. Previous models for single infections, where resistance is a dichotomy, find that treatment rate and the cost of infection to be key factors that contribute to the spread of resistance. By including just one intermediary step before full resistance, in both the human and mosquito population, we demonstrate that although these factors are important, the transmission of resistance is actually best mitigated by controlling the evolution within a host. This result is compounded when one considers that the development to full resistance is actually a continuous process. This model can be used in combination with other models that are investigating this replacement process, and thus one can track how certain factors (such as reducing the selection window) affect the transmission dynamics.

Secondly, provided there is a replacement of sensitive infections with more resistant variants, a disease free equilibrium does not exist. Moreover, a population with only sensitive or partially-resistant infections is not possible. This again highlights the importance of understanding what treatment strategies are the most effective at reducing this replacement rate.

Our work strongly advocates for policies which reduce resistance emerging (or to at least act quickly once it has emerged). However, resistance to malaria treatment has been observed in Africa, yet it has not been estab- 
lished. This may be because the model presented here only considers single infections, and ignores the dynamics within a mosquito. Notwithstanding these additions, the model supports further research into resistance developing within hosts.

\section{Acknowledgements}

The authors wish to thank Prof. Andrew Read, Prof. Sebastian Bonhoeffer and Prof. Ian Hastings for their helpful insights and discussions. This research was funded under Prof. Melissa Penny's Swiss National Science Foundation Professorship PP00P3_170702.

\section{References}

[1] Bell, G. \& MacLean, C. (2017). The Search for 'Evolution-Proof' Antibiotics. Trends in microbiology. https:doi.org10.1016j.tim.2017.11.005.

[2] Boni, M. F., White, N. J. \& Baird, J. K. (2016). The community as the patient in malaria-endemic areas: preempting drug resistance with multiple first-line therapies. PLoS medicine, 13(3), e1001984.

[3] Bushman, M., Morton, L., Duah, N., Quashie, N., Abuaku, B., Koram, K.A., Dimbu, P.R., Plucinski, M., Gutman, J., Lyaruu, P. \& Kachur, S.P. (2016). Within-host competition and drug resistance in the human malaria parasite Plasmodium falciparum. Proceedings of the Royal Soci-

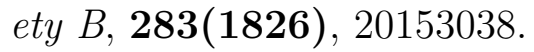

[4] Bushman, M., Antia, R., Udhayakumar, V., \& de Roode, J. C. (2018). Within-host competition can delay evolution of drug resistance in malaria. PLoS biology, 16(8), e2005712.

[5] Chiyaka, C., Garira, W. \& Dube, S. (2007). Transmission model of endemic human malaria in a partially immune population. Mathematical and Computer Modelling, 46(5-6), 806-822.

[6] Chiyaka, C., Garira, W. \& Dube, S. (2009). Effects of treatment and drug resistance on the transmission dynamics of malaria in endemic areas. Theoretical population biology, 75(1), 14-29. 
[7] Day, T., Huijben, S. \& Read, A. F. (2015). Is selection relevant in the evolutionary emergence of drug resistance? Trends in microbiology, 23(3), 126-133.

[8] Day, T., \& Read, A. F. (2016). Does high-dose antimicrobial chemotherapy prevent the evolution of resistance? PLoS computational biology, 12(1), e1004689.

[9] Dietz K, Molineaux L, Thomas A. (1974). A malaria model tested in the African savannah. Bull World Health Org 50, 347-357.

[10] Esteva, L., Gumel, A. B. \& De Leon, C. V. (2009). Qualitative study of transmission dynamics of drug-resistant malaria. Mathematical and Computer Modelling, 50(3-4), 611-630.

[11] Hastings, I. M. (2003). Malaria control and the evolution of drug resistance: an intriguing link. Trends in parasitology, 19(2), 70-73.

[12] Hastings, I. M., \& Hodel, E. M. (2014). Pharmacological considerations in the design of anti-malarial drug combination therapiesis matching half-lives enough? Malaria journal, 13(1), 62.

[13] Hastings, I. M., Watkins, W. M. \& White, N. J. (2002). The evolution of drug-resistant malaria: the role of drug elimination halflife. Philosophical Transactions of the Royal Society B: Biological Sciences, 357(1420), 505-519.

[14] Hastings, I. M., \& Watkins, W. M. (2005). Intensity of malaria transmission and the evolution of drug resistance. Acta Tropica, 94(3), 218-229.

[15] Huijben, S., Sim, D. G., Nelson, W. A. \& Read, A. F. (2011). The fitness of drug-resistant malaria parasites in a rodent model: multiplicity of infection. Journal of evolutionary biology, 24(11), 2410-2422.

[16] Kay, K., \& Hastings, I. M. (2015). Measuring windows of selection for anti-malarial drug treatments. Malaria journal, 14(1), 292.

[17] Koella, J. C., \& Antia, R. (2003). Epidemiological models for the spread of anti-malarial resistance. Malaria Journal, 2(1), 3. 
[18] Kouyos, R. D., Metcalf, C. J. E., Birger, R., Klein, E. Y., zur Wiesch, P. A., Ankomah, P.,Arinaminpathy, N., Bogich, T.L., Bonhoeffer, S., Brower, C. \& Chi-Johnston, G. (2014). The path of least resistance: aggressive or moderate treatment?.Proceedings of the Royal Society of London B: Biological Sciences, 281(1794), 20140566.

[19] Legros, M. \& Bonhoeffer, S. (2016). A combined within-host and between-hosts modelling framework for the evolution of resistance to anti-malarial drugs. Journal of the Royal Society Interface, 13(117), 20160148.

[20] Liechti, J. I., Leventhal, G. E. \& Bonhoeffer, S. (2017). Host population structure impedes reversion to drug sensitivity after discontinuation of treatment. PLoS computational biology, 13(8), e1005704.

[21] Macdonald G. (1957). The epidemiology and control of malaria London, Oxford University Press.

[22] Mackinnon, M. J. (2005). Drug resistance models for malaria. Acta tropica, 94(3), 207-217.

[23] Mandal, S., Sarkar, R. R. \& Sinha, S. (2011). Mathematical models of malaria-a review. Malaria journal, 10(1), 202.

[24] Ménard, D., Khim, N., Beghain, J., Adegnika, A. A., Shafiul-Alam, M., Amodu, O., Rahim-Awab, G., Barnadas, C., Berry, A., Boum, Y. \& Bustos, M. D. (2016). A worldwide map of Plasmodium falciparum K13propeller polymorphisms. New England Journal of Medicine, 374(25), 2453-2464.

[25] Nosten, F. \& White, N. J. (2007). Artemisinin-based combination treatment of falciparum malaria. The American journal of tropical medicine and hygiene, 77(6 Suppl), 181-192.

[26] Read, A. F., Day, T. \& Huijben, S. (2011). The evolution of drug resistance and the curious orthodoxy of aggressive chemotherapy. Proceedings of the National Academy of Sciences, 108(Supplement 2), 10871-10877.

[27] Ross R. (1911). The prevention of malaria, 2nd ed. John Murray, London. 
[28] Ruan, S., Xiao, D. \& Beier, J. C. (2008). On the delayed RossMacdonald model for malaria transmission. Bulletin of mathematical biology, 70(4), 1098-1114.

[29] Sarmah, N. P., Sarma, K., Bhattacharyya, D. R., Sultan, A. A., Bansal, D., Singh, N.,Bharti, P. K., Sehgal, R., Mohapatra, P.K., Parida, P. \& Mahanta, J. (2017). Antifolate drug resistance: Novel mutations and haplotype distribution in dhps and dhfr from Northeast India. Journal of biosciences, 42(4), 531-535.

[30] Shanks, G. D., Edstein, M. D, \& Jacobus, D. (2014). Evolution from double to triple-antimalarial drug combinations. Transactions of The Royal Society of Tropical Medicine and Hygiene, 109(3), 182-188.

[31] Smith, D. L., Battle, K. E., Hay, S. I., Barker, C. M., Scott, T. W. \& McKenzie, F. E. (2012). Ross, Macdonald, and a theory for the dynamics and control of mosquito-transmitted pathogens. PLoS pathogens, 8(4), e1002588.

[32] Tchuenche, J. M., Chiyaka, C., Chan, D., Matthews, A. \& Mayer, G. (2011). A mathematical model for anti-malarial drug resistance. Mathematical medicine and biology: a journal of the IMA, 28(4), 335-355.

[33] Tumwiine, J., Hove-Musekwa, S. D. \& Nyabadza, F. (2014). A mathematical model for the transmission and spread of drug sensitive and resistant malaria strains within a human population. ISRN Biomathematics, 2014.

[34] World Health Organization (2001). Anti-malarial Drug Combination Therapy. Report of a WHO Technical Consultation. Geneva: World Health Organization. WHO/CDS/RBM/2001.35.

[35] Yeung, S., Pongtavornpinyo, W., Hastings, I. M., Mills, A. J. \& White, N. J. (2004). Anti-malarial drug-resistance, artemisinin-based combination therapy, and the contribution of modeling to elucidating policy choices. The American journal of tropical medicine and hygiene, 71(2 suppl), 179-186.

[36] World Health Organization. (2018, August). Status report on artemisinin resistance and ACT efficacy. Retrieved from 
651 http://www.who.int/malaria/publications/atoz/artemisinin-resistance652 august2018/en/

${ }_{653}^{63}$ [37] zur Wiesch, P. A., Kouyos, R., Engelstdter, J., Regoes, R. R. \& Bonhoeffer, S. (2011). Population biological principles of drug-resistance

655 evolution in infectious diseases. The Lancet infectious diseases, 11(3), $656 \quad 236-247$. 


\section{Appendix A. The reproductive numbers}

The disease free equilibrium point is when the human population is

$$
I_{\mathcal{S}}=I_{\mathcal{P}}=I_{\mathcal{R}}=T_{\mathcal{S}}=T_{\mathcal{P}}=T_{\mathcal{R}}=0 \text { and } S=N
$$

and the mosquito population is

$$
\hat{E}_{\mathcal{S}}=\hat{E}_{\mathcal{P}}=\hat{E}_{\mathcal{R}}=I_{\mathcal{S}}=I_{\mathcal{P}}=I_{\mathcal{R}}=0 \text { and } \hat{S}=\hat{N}=1
$$

Consider a single newly infectious mosquito with any class of infection. At time $t$ this mosquito has a probability $e^{-\hat{u} t}$ of surviving its infectious period, and infects humans at a rate $a b_{j} m S / N$. Hence the total number of humans who become infectious, from each class, due to this mosquito during its entire infectious period is

$$
\begin{aligned}
R_{\hat{I}_{j} \rightarrow I_{j}} & =a b_{j} m \frac{S}{N} \int_{\mathcal{S}}^{\infty} e^{-\hat{u} t} \mathrm{~d} t \\
& =\frac{a b_{j} m}{\hat{\mu}}
\end{aligned}
$$

A similar process is used to derive the total number of mosquitoes who become infectious from a human during his/her entire infectious period. However, there are several different routes the infection can take, see (9)-(11). These different routes are detailed below.

Appendix A.1. Equation (9): $R_{\mathcal{S} \rightarrow \mathcal{S}}=\left(R_{I_{\mathcal{S}} \rightarrow \hat{I}_{\mathcal{S}}}+R_{I_{\mathcal{S}} \rightarrow T_{\mathcal{S}} \rightarrow \hat{I}_{\mathcal{S}}}\right) R_{\hat{I}_{\mathcal{S}} \rightarrow I_{\mathcal{S}}}$

The expected number of mosquitoes who become infectious with a sensitive infection, from one human with this infection who is not treated, is

$$
\begin{aligned}
R_{I_{\mathcal{S}} \rightarrow \hat{I}_{\mathcal{S}}} & =a c_{\mathcal{S}} e^{-\hat{\mu} \hat{\tau}} \int_{\mathcal{S}}^{\infty} e^{-\left(r_{I}+r_{x}+\alpha\right) t} \mathrm{~d} t \\
& =a c_{\mathcal{S}} \frac{1}{r_{I}+r_{x}+\alpha} e^{-\hat{\mu} \hat{\tau}} .
\end{aligned}
$$

And if the human is treated, which occurs at a rate $r_{x}$, the expected number of infectious mosquitoes is,

$$
\begin{aligned}
R_{I_{\mathcal{S}} \rightarrow T_{\mathcal{S}} \rightarrow \hat{I}_{\mathcal{S}}} & =a c_{\mathcal{S}} e^{-\hat{\mu} \hat{\tau}} r_{x} \int_{\mathcal{S}}^{\infty} \int_{\mathcal{S}}^{\infty} e^{-\left(r_{I}+r_{x}+\alpha\right) u} e^{-\left(r_{T_{\mathcal{S}}}+\phi+\mu\right) t} \mathrm{~d} u \mathrm{~d} t \\
& =a c_{\mathcal{S}} \frac{r_{x}}{\left(r_{I}+r_{x}+\alpha\right)\left(r_{T_{\mathcal{S}}}+\phi+\mu\right)} e^{-\hat{\mu} \hat{\tau}} .
\end{aligned}
$$


Combining with (A.1) gives the total number of secondary sensitive infections from one human infected with a sensitive infection,

$$
R_{\mathcal{S} \rightarrow \mathcal{S}}=\frac{a^{2} b_{\mathcal{S}} c_{\mathcal{S}} m}{\hat{\mu}}\left[\frac{r_{T_{\mathcal{S}}}+r_{x}+\phi+\mu}{\left(r_{I}+r_{x}+\alpha\right)\left(r_{T_{\mathcal{S}}}+\phi+\mu\right)}\right] e^{-\hat{\mu} \hat{\tau}} .
$$

Appendix A.2. Equation (10): $R_{\mathcal{P} \rightarrow \mathcal{P}}=\left(R_{I_{\mathcal{P}} \rightarrow \hat{I}_{\mathcal{P}}}+R_{I_{\mathcal{P}} \rightarrow T_{\mathcal{P}} \rightarrow \hat{I}_{\mathcal{P}}}\right) R_{\hat{I}_{\mathcal{P}} \rightarrow I_{\mathcal{P}}}$

The expected number of mosquitoes who become infectious with the partially-resistant infection, from one human with this infection who is not treated, is the parallel to (A.2). Similarly, if the human is treated, which occurs at a rate $r_{x}$, the expected number of infectious mosquitoes is the parallel to (A.3). Therefore, the total number of secondary infected humans, with a partially-resistant infection, from one human infected with a partiallyresistant infection, is

$$
R_{\mathcal{P} \rightarrow \mathcal{P}}=\frac{a^{2} b_{\mathcal{P}} c_{\mathcal{P}} m}{\hat{\mu}}\left[\frac{r_{T_{\mathcal{P}}}+r_{x}+\phi+\mu}{\left(r_{I}+r_{x}+\alpha\right)\left(r_{T_{\mathcal{P}}}+\phi+\mu\right)}\right] e^{-\hat{\mu} \hat{\tau}}
$$

\section{Appendix A.3. Equation (11): $R_{\mathcal{R} \rightarrow \mathcal{R}}=\left(R_{I_{\mathcal{R}} \rightarrow \hat{I}_{\mathcal{R}}}+R_{I_{\mathcal{R}} \rightarrow T_{\mathcal{R}} \rightarrow \hat{I}_{\mathcal{R}}}\right) R_{\hat{I}_{\mathcal{R}} \rightarrow I_{\mathcal{R}}}$}

The expected number of mosquitoes who become infectious with the fullyresistant infection, from one human with this infection who is not treated, is the same as (A.2). However, if the human is treated, which occurs at a rate $r_{x}$, the expected number of infectious mosquitoes is

$$
\begin{aligned}
R_{I_{\mathcal{R}} \rightarrow T_{\mathcal{R}} \rightarrow \hat{I}_{\mathcal{R}}} & =a c_{\mathcal{P}} e^{-\hat{\mu} \hat{\tau}} r_{x} \int_{\mathcal{S}}^{\infty} \int_{\mathcal{S}}^{\infty} e^{-\left(r_{I}+r_{x}+\alpha\right) u} e^{-\left(r_{I}+\alpha\right) t} \mathrm{~d} u \mathrm{~d} t \\
& =a c_{\mathcal{P}} \frac{r_{x}}{\left(r_{I}+r_{x}+\alpha\right)\left(r_{I}+\alpha\right)} e^{-\hat{\mu} \hat{\tau}} .
\end{aligned}
$$

Combining with (A.1) gives the total number of secondary infected humans, with a fully-resistant infection, from one human infected with a fully-resistant infection,

$$
R_{\mathcal{R} \rightarrow \mathcal{R}}=\frac{a^{2} b_{\mathcal{R}} c_{\mathcal{P}} m}{\hat{\mu}} \frac{1}{r_{I}+\alpha} e^{-\left(r_{A}+\mu\right) \tau} e^{-\hat{\mu} \hat{\tau}}
$$

\section{Appendix A.4. Equation (12): $R_{I_{\mathcal{S}} \rightarrow T_{\mathcal{S}} \rightarrow T_{\mathcal{P}} \rightarrow \hat{I}_{\mathcal{P}}} R_{\hat{I}_{\mathcal{P}} \rightarrow I_{\mathcal{P}}}$}

A human infected with a sensitive infection may infect a mosquito with partially-resistant infection. This human would be treated at a rate $r_{x}$, and 
become partially-resistant at rate $\phi$, giving,

$$
\begin{aligned}
R_{I_{\mathcal{S}} \rightarrow T_{\mathcal{S}} \rightarrow T_{\mathcal{P}} \rightarrow \hat{I}_{\mathcal{P}}}= & a c_{\mathcal{S}} e^{-\hat{\mu} \hat{\tau}} r_{x} \phi \int_{\mathcal{S}}^{\infty} \int_{\mathcal{S}}^{\infty} \int_{\mathcal{S}}^{\infty} e^{-\left(r_{I}+r_{x}+\alpha\right) v} e^{-\left(r_{T}+\phi+\mu\right) u} \\
& e^{-\left(r_{T}+\phi+\mu\right) t} \mathrm{~d} u \mathrm{~d} v \mathrm{~d} t \\
= & \frac{a c_{\mathcal{S}} \mu}{\lambda} \frac{r_{x} \phi}{\left(r_{I}+r_{x}+\alpha\right)\left(r_{T_{\mathcal{S}}}+\phi+\mu\right)\left(r_{T_{\mathcal{P}}}+\phi+\mu\right)} e^{-\hat{\mu} \hat{\tau}} .
\end{aligned}
$$

Combining with (A.1) gives the total number of secondary infected humans, with a partially-resistant infection, from one human infected with a sensitive infection,

$$
R_{\mathcal{S} \rightarrow \mathcal{P}}=\frac{a^{2} b_{\mathcal{P}} c_{\mathcal{S}} m}{\hat{\mu}}\left[\frac{r_{x} \phi}{\left(r_{I}+r_{x}+\alpha\right)\left(r_{T_{\mathcal{S}}}+\phi+\mu\right)\left(r_{T_{\mathcal{P}}}+\phi+\mu\right)}\right] e^{-\hat{\mu} \hat{\tau}} .
$$

Appendix A.5. Equation (13): $R_{I_{\mathcal{P}} \rightarrow T_{\mathcal{P}} \rightarrow T_{\mathcal{R}} \rightarrow \hat{I}_{\mathcal{R}}} R_{\hat{I}_{\mathcal{R}} \rightarrow I_{\mathcal{R}}}$

A human infected with a sensitive infection may infect a mosquito with a partially-resistant infections. This human would be treated at a rate $r_{x}$, and become partially-resistant at rate $\phi$, giving,

$$
\begin{aligned}
R_{I_{\mathcal{P}} \rightarrow T_{\mathcal{P}} \rightarrow T_{\mathcal{R}} \rightarrow \hat{I}_{\mathcal{R}}}= & a c_{\mathcal{P}} e^{-\hat{\mu} \hat{\tau}} r_{x} \phi \int_{\mathcal{S}}^{\infty} \int_{\mathcal{S}}^{\infty} \int_{\mathcal{S}}^{\infty} e^{-\left(r_{I}+r_{x}+\alpha\right) v} e^{-\left(r_{T}+\phi+\mu\right) u} \\
& e^{-\left(r_{I}+\alpha\right) t} \mathrm{~d} u \mathrm{~d} v \mathrm{~d} t \\
= & a c_{\mathcal{P}} \frac{r_{x} \phi}{\left(r_{I}+r_{x}+\alpha\right)\left(r_{T_{\mathcal{P}}}+\phi+\mu\right)\left(r_{I}+\alpha\right)} e^{-\hat{\mu} \hat{\tau}} .
\end{aligned}
$$

Combining with (A.1) gives the total number of secondary infected humans, with a fully-resistant infection, from one human infected with a partiallyresistant infection,

$$
R_{\mathcal{P} \rightarrow \mathcal{R}}=\frac{a^{2} b_{\mathcal{R}} c_{\mathcal{P}} m}{\hat{\mu}}\left[\frac{r_{x} \phi}{\left(r_{I}+r_{x}+\alpha\right)\left(r_{T_{\mathcal{P}}}+\phi+\mu\right)\left(r_{I}+\alpha\right)}\right] e^{-\hat{\mu} \hat{\tau}} .
$$

Appendix A.6. Equation (14): $R_{I_{\mathcal{S}} \rightarrow T_{\mathcal{S}} \rightarrow T_{\mathcal{P}} \rightarrow T_{\mathcal{R}} \rightarrow \hat{I}_{\mathcal{R}}} R_{\hat{I}_{\mathcal{R}} \rightarrow I_{\mathcal{R}}}$

A human infected with a sensitive infection may infect a mosquito with partially-resistant infection. This human would be treated at a rate $r_{x}$, become partially-resistant at rate $\phi$, and then fully-resistant at rate $\phi$, giving,

$$
\begin{aligned}
R_{I_{\mathcal{S}} \rightarrow T_{\mathcal{S}} \rightarrow T_{\mathcal{P}} \rightarrow T_{\mathcal{R}} \rightarrow \hat{I}_{\mathcal{R}}}= & a c_{\mathcal{S}} e^{-\hat{\mu} \hat{\tau}} r_{x} \phi^{2} \int_{\mathcal{S}}^{\infty} \int_{\mathcal{S}}^{\infty} \int_{\mathcal{S}}^{\infty} \int_{\mathcal{S}}^{\infty} e^{-\left(r_{I}+r_{x}+\alpha\right) w} e^{-\left(r_{T}+\phi+\mu\right) v} \\
& e^{-\left(r_{T}+\phi+\mu\right) u} e^{-\left(r_{I}+\alpha\right) t} \mathrm{~d} w \mathrm{~d} u \mathrm{~d} v \mathrm{~d} t \\
= & a c_{\mathcal{S}} \frac{r_{x} \phi^{2}}{\left(r_{I}+r_{x}+\alpha\right)\left(r_{T_{\mathcal{S}}}+\phi+\mu\right)\left(r_{T_{\mathcal{P}}}+\phi+\mu\right)\left(r_{I}+\alpha\right)} e^{-\hat{\mu} \hat{\tau}} .
\end{aligned}
$$


Combining with (A.1) gives the total number of secondary infected humans, with a fully-resistant infection, from one human infected with a sensitive infection,

$$
R_{\mathcal{S} \rightarrow \mathcal{R}}=\frac{a^{2} b_{\mathcal{R}} c_{\mathcal{S}} m}{\hat{\mu}}\left[\frac{r_{x} \phi^{2}}{\left(r_{I}+r_{x}+\alpha\right)\left(r_{T_{\mathcal{S}}}+\phi+\mu\right)\left(r_{T_{\mathcal{P}}}+\phi+\mu\right)\left(r_{I}+\alpha\right)}\right] e^{-\hat{\mu} \hat{\tau}}
$$

\section{Appendix B. Day and Read 2016}

The probability of resistance emerging is approximately equal to

$$
\phi=1-e^{-H(c)}
$$

where $H(c)$ is the resistant hazard,

$$
H(c)=D(c)+S(c)
$$

The quantity $D(c)$ is the de novo hazard,

$$
D(c)=\int_{\mathcal{S}}^{a} \lambda[p(s ; c), c] \pi[x(s ; c), c] \mathrm{d} s .
$$

71 Is is comprised of the integral of the product of $\lambda[p(s ; c), c]$, the rate at which resistant mutants appear at time $s$ after the start of treatment, and $\pi[x(s ; c), c]$, the probability of escape of any such mutant.

The quantity $S(c)$ is the standing hazard - the hazard due to a standing population of $n$ resistant microbes that are already present at the beginning of treatment,

$$
S(c)=-n \ln (1-\pi[x(0 ; c), c])
$$

